\title{
Perspective
}

\section{While I Still Remember: 30 Years of Alzheimer's Disease Research}

\author{
Thomas B. Shea* \\ Laboratory for Neuroscience, Department of Biological Sciences, UMass Lowell, Lowell, MA, USA
}

Accepted 10 September 2017

\begin{abstract}
Turns out I have been a major contributor to the Journal of Alzheimer's Disease over its 20-year history. As such, I was invited to provide a review of my work over the years. What follows is a retrospective of how the Alzheimer-related research of a Ph.D. (i.e., not an M.D.) transitioned from basic to clinical, and moved from bench to bedside and back again. I have included some of the more humorous and poignant twists along the way that some older players may find familiar and I hope might inspire some younger players to hang in there.
\end{abstract}

Keywords: Environmental toxins, methionine cycle, nutrition, oxidative damage, translational studies, traumatic brain injury

\section{THE ROLE OF JAD}

I have been privileged to witness the Journal of Alzheimer's Disease (JAD) morph over the last 20 years from a grass-roots "by the people" journal into a, if not the, premier journal in which to publish and to locate studies on all aspects of neuronal homeostasis and perturbation related to dementia. As many journals rise in prominence, and of necessity become more selective in which manuscripts are accepted for publication, the editorial board often takes on a "stuffy/holier-than-thou" stance. This has not happened with JAD, which has remained a major forum for all things Alzheimer's disease (AD). I recall an editor's meeting where founder George Perry said, when JAD's impact factor approached 5, he was of course pleased but did not want it to go higher than that, since such an approach imposes selection of articles that will continue to increase the impact factor. The flip side of that is the rejection of articles, regardless of quality, that will likely not be widely accepted should they challenge accepted views.

\footnotetext{
*Correspondence to: Thomas B. Shea, Ph.D., Professor \& Director, Laboratory for Neuroscience, Department of Biological Sciences, UMass Lowell, Lowell, MA 01854, USA. Tel.: +1 978 9342881; Fax:+1978934 3044; E-mail:Thomas_Shea@uml.edu.
}

JAD allowed novel articles, novel small approaches, reactionary and revolutionary ideas, and articles not taking part in the endless " $\mathrm{A} \beta$ versus tau as the singular cause of AD" battles. This was in no small way due to the originator and editor, George Perry, himself a wellspring of reactionary and revolutionary ideas, and the late Mark Smith, who advanced the then heretical hypothesis that increased $\mathrm{A} \beta$ during neurodegeneration was an attempt at neuroprotection due to antecedent problems such as oxidative damage. Mark drew the outstanding analogy of the damage caused by $A \beta$ to that of firemen arriving at one's home: they may break down doors, chop through walls, and flood the area, but in doing do they just might put out the fire and save the home [1]. I found and still find solace in the line of reasoning that, should this hypothesis be correct, what we witness by definition are only the $A \beta$ failures; if a satisfactory neuroprotective response were indeed mounted by $A \beta$, no clinical signs would manifest.

This is not to imply that articles early on in JAD's history were inferior, but rather that they sometimes explored novel areas and consequently, were small in size. "You gotta start somewhere," seemed uniquely permissible for a submission to JAD. Indeed, how can 
one obtain mega-funding for an area with virtually (or literally) no existing studies, however preliminary they may be. This is analogous to the "experience needed" for employment, leaving an entry-level applicant unable to gain any experience since he/she cannot secure employment.

The ability to publish somewhat radical ideas gave my colleagues and I the hand-hold needed to develop and submit a proposal to the Alzheimer's Association in the $1990 \mathrm{~s}$ on the potential role of homocysteine in dementia-related neurodegeneration. There were and are a number of good nutrition-based journals. However, JAD provided a unique forum for connection of nutrition with $\mathrm{AD}$ neuropathology. Among the reviews of this unsuccessful proposal was the criticism that "There is no evidence for a role for homocysteine." Notably, 3 years later, the reviews of our second revision (i.e., 3rd attempt) of this proposal included the statement that "Everyone knows that!" We just made it. Without ongoing acceptance by JAD during these times, my group might have abandoned this line of research, which after nearly 20 years led to successful phase II clinical studies, themselves published in JAD. Tracking the papers that I have handled as editor has shown me that this is not a unique story, but rather a consistent role played by JAD in allowing novel ideas, and young investigators, to flourish.

The "shareholder" approach of providing an author a term as an Associate Editor was, at least to me as a young faculty member, an important feeling of camaraderie and spurred on word-of-mouth/email solicitation of colleagues' submissions. I find it poignant that, due to number of publications, referrals, and service as both Senior and Associate Editor, I have an appointment as Associate Editor until 2037, which is well into my 80s. I stand the mind-boggling possibility of presenting AD while still and editor for JAD, hence the title "While I still remember..."

Here's to another 20 years for JAD, more successful than the first 20 ! And I hope I am aware.

\section{TIMING IS EVERYTHING}

My forays into neurodegeneration began in the laboratory of Ralph Nixon at McLean Hospital. My studies began with studying axon outgrowth in cultured neuronal systems. However, the Nixon laboratory also a number of ongoing AD-related studies, and in short time, I would add an extra set or two of cultures to my experiments where I would perturb one or more developmental systems. It quickly became apparent that while slight activation or inhibition of kinase or protease systems stimulated normal development, overactivation or severe inhibition of the same systems promoted "AD-like" mislocalization and hyperphosphorylation of cytoskeletal proteins including tau. I ended up with a dual approach for most experiments, where untreated or mildly stimulated control cultures would provide indications of normal development, yet would also serve as controls for hyperactivation of alternate cultures. My studies were consequently funded both by the National Science Foundation for normal outgrowth and the Alzheimer's Association and the like for neurodegenerative studies. This overlapping approach minimized the need for cultures and I was able to remain productive on limited resources.

We were busy manipulating a laundry list of kinase and phosphatase pathways and monitoring their impact on neurofilaments, MAP2, and tau, and were unaware that among these were "the" tau kinases, which turned out to be the none other than the ubiquitous glycogen synthase kinase $3 \beta$ (GSK3 $\beta$ ), cyclin-dependent kinase 5 (cdk5), and pp44-45 mitogen-activated kinase (MAPk). We and others also found that tau underwent sequential phosphorylation, and that the order of kinase action was critical in generation of AD-like phosphorylation [2-4]. This "unique critical order" turned out to be nothing more than priming of the consensus sequences for the proline-directed GSK3 $\beta$ by nonproline directed kinases such as protein kinase $\mathrm{C}$ (PKC) - yet another common player in many cellular processes. Modest activation of GSK3 $\beta$, cdk5, or MAPk promoted axon outgrowth and segregation within axons of phospho-neurofilaments and phospho-tau characteristic of normal development. However, a bit more activation instead induced accumulation of phospho-isoforms of both within perikarya. During this time, we also noted that slight inhibition of the calcium-activated protease calpain promoted axonal outgrowth, but activation instead generated classical "AD-like phosphorylation" [5-7]. Moreover, these kinase and protease systems were intimately related in that the impact of calpain on both normal developmental and neurodegenerative was mediated by modulation of PKC activity [8], which in turn modulated GSK3 $\beta$ activity, which in turn modulated axonal transport, including that of neurofilaments and tau.

Additional studies added timing as a factor. We saw that simple withdrawal of serum from culture medium promoted differentiation, leading us to carry 
out a study showing that differentiation was a default pathway held in check in neuroblasts pending developmental withdrawal from the mitotic cycle [9]. Secondary glial-derived growth factors that were then essential for axonal outgrowth and nervous system development promoted neurodegeneration if secreted at the wrong time [10-13]. Similarly, while $A \beta$ was neurotoxic to established neuronal cultures, it was actually beneficial to undifferentiated neurons or those in the earliest stages of differentiation [14]. I remember thinking that tweaking the same systems spelled life or death. These findings led us to embrace the notion put forth later from others that subtle alteration of "housekeeping" systems could spell the difference between homeostasis and degeneration.

\section{FROM CHEMICAL TO NATURAL APPROACHES}

In the mid 1990 s, I relocated my growing research operation to UMass Lowell, which provided a number of advantages. The most important of these was that I met faculty who specialized in nutritional research, who noted that some of the systems and pathways I had been studying were amenable to nutritional intervention. Based on this suggestion, I incorporated a few nutritional as well as pharmacological compounds in our ongoing experiments. Using vitamin E, which quenched oxidative damage but did not alter kinase activity, we were able to discern that not only did tau hyperphosphorylation not correlate with neurodegeneration in cultured cortical neurons, but moreover that, while $A \beta$ increased oxidative stress and phosphorylation of tau, neurodegeneration induced by $\mathrm{A} \beta$ was due entirely to oxidative stress $[15,16]$. These observations sparked what would be a $20+$ year collaboration and eventually lead to a vitamin/nutraceutical formulation effective against multiple biochemical/histological biomarkers of $\mathrm{AD}$ neuropathology in laboratory studies and effective against cognitive and behavioral decline for individuals diagnosed with $\mathrm{AD}$ or mild cognitive impairment (MCI) in clinical studies. Recalling Mark Smith's hypothesis that increased $A \beta$ may be a reaction to neurotrauma rather than a cause, these results also steered my focus upstream of $A \beta$.

A second major advantage of UMass Lowell was that at the time (late 1990s), there was no per diem charge for mice. I therefore had the opportunity to maintain large colonies of multiple transgenic mice, with multiple breeding pairs, therefore at various ages. And the last major advantage of relocating to UMass Lowell was what seemed like an endless stream of graduate students to whom "Alzheimer's-related research" was more attractive than other more basic research. Overlapping experiments (where untreated controls tracked normal neuronal development), inexpensive reagents (vitamins and nutraceuticals), unlimited mice, and many eager graduate students allowed us to embark on a series of studies of nutritional supplementation or deprivation with normal and transgenic mouse lines.

Nutritional expert Eugene Rogers proposed examining the impact of one-carbon metabolism, errors in which fostered accumulation of homocysteine. I was completely ignorant of this pathway, also known as the Methionine Cycle. His interests lay in basic research. But new research pointed toward homocysteine as a potent neurotoxin, and when Eugene pointed out that a component of this cycle (S-adenosyl methionine, or "SAMe") was the major methyl donor (including methylation of DNA), I saw this as an excellent means to position nutrition against genetics in AD neuropathology. We maintained cohorts of adult mice ( 9 months of age) lacking murine Apolipoprotein E (ApoE-/-) mice, along with normal $(\mathrm{ApoE}+/+)$ on a standard mouse diet and that diet lacking folate and vitamin E for one month [17]. We found overall oxidative damage to brain tissue, more so than liver (as an index of systemic damage) exclusively for ApoE-/- mice lacking folate and vitamin E. Surprisingly, we also observed cognitive impairment for ApoE-/- mice lacking these vitamins [18]. Normal mice displayed no increase in oxidative damage nor cognitive decline when deprived of folate and vitamin E, and ApoE-/- mice displayed no oxidative damage nor cognitive decline when provided folate and vitamin $\mathrm{E}$. The power of these findings was that a genetic predisposition for oxidative damage to brain tissue and accompanying cognitive decline could be overcome by simple supplementation.

We then further manipulated the methionine cycle. In addition to folate and vitamin E, we supplemented additional cohorts of mice with precursors and compounds that were part of the methionine cycle such as B12, SAMe and N-acetylcysteine (NAC), and Acetyl-L-carnitine (ALCAR) to protect mitochondria and maintain ATP levels. In doing so, we increased glutathione production and lowered oxidative species in brain tissue. Notably, when ApoE-/- mice were deprived of folate and vitamin E, they displayed significantly higher levels of glutathione, but still showed increased oxidative 
damage. We initially thought that generation of excess glutathione was a failed attempt to quench oxidative species [19-21]. However, because of the large number of cohorts on multiple diets, we had ApoE-I- mice deprived of folate and vitamin E but supplemented with SAMe. These mice displayed normal glutathione levels, yet reduced oxidative species. We essentially stumbled upon the finding that SAMe is an obligate cofactor for glutathione bioavailability. Specifically, glutathione-S-transferase requires SAMe for transient methylation of oxidative species for subsequent reduction by glutathione [22, 23]. Further study revealed that the expression and activity of multiple additional genes of the methionine cycle were altered [24]. Similar findings were observed when we compared mice expressing human ApoE3 versus ApoE4 [25]. Interestingly, normal aged mice (2-2.5 years old) displayed biochemical and cognitive impairments similar to those of 9-month old ApoE-/- mice [26], leading us to hypothesize that a genetic deficiency in oxidative buffering capacity could play out as "advanced aging."

We also manipulated the methionine cycle in neuronal culture studies, which revealed aberrant calcium influx, by multiple pathways, to underlie multiple neurodegenerative events including increased oxidative damage, glutamate toxicity, apoptosis, and homocysteine generation, the latter of which potentiated all of the above phenomena $[15,16$, 27-31]. Impairment of the methionine cycle fostered aberrant calcium influx by multiple methods, including a reduction of methylation of channels as well as overactivation of phosphorylation by decreased methylation of phosphatases [32, 33].

We also cultured cortical neurons in petri dishes containing multi-electrode arrays, which allowed us to monitor synaptic signaling. Supplementation to bolster the methionine cycle increased complex signaling characteristic of healthy neurons, while folate and vitamin $\mathrm{E}$ deprivation prompted disorganized signaling [34].

In addition to the above more general effects, our studies also demonstrated that bolstering the methionine cycle was beneficial for many $\mathrm{AD}$-specific biomarkers, including prevention of PS-1 overexpression by methylation of the gene, prevention of betaand gamma-secretase activity, reduction of overall $\mathrm{A} \beta$ levels (including extracellular deposits) and toxicity, reduction of phosphorylated tau, maintenance of acetylcholine levels, improvement of cognitive performance and reduction of aggression. Notably, all of the above effects were deleteriously affected by impairment of the cycle by folate and vitamin E deprivation (see references in $[35,36]$ ).

These studies collectively demonstrated that nutrition was pivotal not only for general neuronal health, but for modulation of multiple aspects of AD-like neuropathology.

\section{FROM THE BENCH TO THE CLINIC}

Overall, we conducted $>40$ studies with supplementation of cultures and various mouse models, which lead to a combinatorial formulation containing ingredients that bolstered the methionine cycle (Folate, B12, SAMe, NAC) along with vitamin E as a first-line of antioxidant defense and ALCAR to maintain ATP.

We made a small, open-label foray with this "Nutraceutical Formulation" with communitydwelling individuals diagnosed with mild to moderate $\mathrm{AD}$, relying on the expertise of Ruth Remington from UMass Lowell's Department of Nursing and Dr. James Paskevitz of the Dementia Clinic at UMass Memorial Medical Center (Worcester, MA) and internal funding from UMass Lowell. Participants demonstrated statistical improvement in cognitive performance (Clock-Drawing test and Dementia Rating scale), while caregivers reported statistical improvement in mood/behavior (Neuropsychiatric Inventory) and maintenance of daily function (Activities of Daily Living) during the 12-month trial. Caregivers requested that the trial be extended. Institutional Review Board restrictions could not allow continued cognitive testing without a new application (since this required visitations and interaction with participants). However, a rapid minor amendment allowed us to continue to deliver the formulation and for caregivers to continue their assessment Neuropsychiatric Inventory and Activities of Daily Living assessments for an additional 16 months. Participants maintained their performance throughout this extension, resulting in efficacy for a total of 2 years and 2 months [37].

A second 9-month study with nursing home residents diagnosed with moderate-severe $\mathrm{AD}$ demonstrated a slowing of decline in cognitive performance and improvement in mood/behavior for those receiving the formulation versus placebo [38].

We conducted a series of trials with communitydwelling participants with no known or suspected cognitive difficulties. Participants improved statistically by $100 \%$ in short-term word recollection 
(California Verbal Learning Test II) and by $20 \%$ in executive function (Trails Test A \& B) within 3 months. All participants were then provided with the formulation under open-label conditions. Participants originally receiving placebo improved within 3 months to the same extent as those originally receiving the formulation for 3 months, while those originally receiving the formulation continued to improve. We then removed the formulation from all participants for 3 months, after which time they had declined to their respective baseline performance. Participants then resumed the formulation for 3 months, after which they once again demonstrated improvement to the same extent as after their first 3 months of receiving the formulation. Based on the extent of improvement within just 3 months, we conducted an additional randomized trial with a second cohort, in which participants receiving the formulation improved statistically in executive function within just 2 weeks, and those receiving the placebo did not improve until crossed-ever to the formulation for 2 weeks [39].

Based on these results, the formulation was awarded a US patent and we were able to compete successfully for funding from the national Alzheimer's Association for phase II studies. These studies, all of which appeared in JAD, confirmed cognitive and behavioral improvement for individuals with $\mathrm{AD}$ and cognitive improvement for those diagnosed with MCI versus those receiving placebo. Participants originally randomized to placebo also demonstrated improvement following cross-over to the formulation [40-42]. Individuals diagnosed with AD who had previously begun consuming the formulation on their own requested enrollment in these studies. They were allowed to do so under open-label conditions, since randomization would inappropriately force cessation of consumption by some of these individuals. These participants sustained cognitive and behavioral performance over the course of 12 months, during which time historical placebos (from other publications) and placebo cohorts from our own studies had declined [43].

These studies demonstrated that nutritional intervention can be effective for cognition, mood, and function for individuals prior to cognitive decline, individuals diagnosed with $\mathrm{MCI}$, and for all stages of AD.

Due to funding and personnel limitations as well as the relative novelty of this nutraceutical formulation, our studies were confined to cognitive and behavioral performance. However, since laboratory studies indicate that this formulation reduced multiple $\mathrm{AD}$ hallmarks including maintenance of neurotransmitters, reduction of intracellular $A \beta$, extracellular $\mathrm{A} \beta$-reactive plaques, and hyperphosphorylated tau $[26,44]$, it is possible that the formulation could have a similar impact for individuals with AD. Also of interest would be a prospective longitudinal study to determine whether or not initiation of consumption of this formulation could delay a diagnosis of AD. This is a reasonable possibility since individuals without cognitive difficulties and those with MCI demonstrated improved cognitive performance for at least 12 months. In addition, some individuals in our phase II studies that had been diagnosed with $\mathrm{AD}$ prior to baseline improved to the extent that their scores were consistent with normal performance rather than that anticipated for individuals with AD.

Notably, the extent of improvement observed in the Neuropsychiatric Inventory in both phase I and phase II studies was statistically identical to that observed for donepezil in another study [37]. We by no means suggest that this formulation is a substitute for any prescribed pharmacological agent, but rather make this point to highlight that nutritional intervention can have a significant impact. Since donepezil is an acetylcholinesterase inhibitor, which functions by preserving existing acetylcholine levels, and laboratory studies indicate that SAMe (a component of our formulation) maintains acetylcholine production [26], donepezil and this formulation may be more effective in combination.

\section{IT STARTED LONG AGO}

Many studies support the notion that cognitive decline has begun in individuals that will develop $\mathrm{AD}$ years to decades before clinical manifestation. If this is the case (and the data suggest it is), then at least one of the mitigating factors must, by definition, have occurred at the start of this antecedent interval, and must have been sufficiently benign to remain unnoticed and avoid triggering intervention. One such example was co-treatment of cultured neurons with homocysteine and $A \beta$, each at concentrations $(10 \mu \mathrm{M})$ that alone provoked no detectable deleterious effect, yet together invoked oxidative damage, calcium perturbations, and hyperphosphorylation of tau to a greater extent than observed with $20 \mu \mathrm{M}$ of either agent alone [45].

Along these lines, within the past few years we have taken advantage of our MEA system to 
monitor subtle changes in synaptic signaling, with the rationale that any such changes could precede overt cognitive impairment or neurodegenerative hallmarks. Indeed, we observed reversible inhibition of complex signaling with exogenously-applied low concentrations of $A \beta$ that did not invoke oxidative damage or calcium perturbations characteristic of higher concentrations [46]. We also observed that subcytotoxic concentrations of iron and $A \beta$ had an additive suppression of complex signaling, and further that this deleterious impact was alleviated by concentrations of zinc that had no detectable impact alone [47].

Such mild delay or suppression in synaptic activity may not be detected during everyday life or even during a standard cognitive test, but may spell all the difference during a critical time-dependent situation such as the rapid reaction and decision-making required to avoid a traffic accident.

\section{WHAT ARE SOME UPSTREAM EVENTS CONTRIBUTING TO AD?}

$\mathrm{AD}$ has an apparent multifactorial etiology encompassing nutritional, genetic, and environmental risk factors, none of which is sufficient to account for all cases [48, 49]. Convergence of these factors may be required for clinical manifestation [50, 51]. Genetic predispositions may be latent pending agerelated decline in nutrition [52]. We have already discussed our work with nutrition, and much of that work included positioning of nutrition with/against genetics, including examples of how supplementation can overcome the deleterious impact of alleles that promote $\mathrm{AD}$-like neurodegeneration. However, the contribution of genetics is exceptionally complex. While some alleles and mutations have been clearly identified as causal/contributory, there is a less obvious facet of genetics, which is what I like to refer to as "permissive alleles." We had a very protracted series of revisions for a particular "position paper," ultimately published in JAD, which advanced the possibility that the E3 allele of ApoE was in a sense a risk factor for AD [53]. The focus of this fight was to underscore that a host of alleles of key genes (including those yet to be recognized) may not directly promote $\mathrm{AD}$, but may be permissive in the sense that they do not provide critical neuroprotection afforded by other alleles.

To try and quantify the impact of environmental enrichment and social interaction, we resorted to our mouse models. Following systems set in place by others, we examined the impact of environmental enrichment versus nutritional supplementation in mice by housing mice either individually or in groups under "standard" (a $173 \mathrm{~cm}^{2}$ enclosure layered with pine chip bedding) or "enriched" (a $388 \mathrm{~cm}^{2}$ enclosure with chewable nesting material for burrowing and plastic toys rotated weekly) environments, and provided them with either a complete diet or a diet lacking folate and vitamin E. Among all groups, mice receiving the complete diet housed in the enriched environment displayed superior performance in $\mathrm{Y}$ maze navigation [54]. In a separate study, a combination of environmental enrichment and housing of mice in groups of 3 provided an additive boost to cognitive performance in normal adult mice, as compared to mice maintained in a standard environment and/or in isolation [55].

These findings collectively indicate that environmental, social, and dietary enrichment can have a positive impact on cognitive performance. We note that so-called enriched environments are in fact not at all enriched from the perspective of the laboratory animal. Providing mice with a larger enclosure containing a few novel objects for exploration and the possibility of digging a nest actually only serves to approximate a natural environment; a standard mouse cage with no objects and a thin layer of standard bedding is actually an extremely deprived environment, analogous to an individual living alone in a bleak tiny apartment or room in a nursing home and eating poorly. Housing mice in isolation is also unnatural. We could therefore re-evaluate such studies from the "reverse" viewpoint of the impact of a deprived environment, lack of social interaction, and poor nutrition on cognitive decline, rather than what may be the less accurate viewpoint of a so-called enriched environment, social interaction, and nutritional supplementation on cognitive improvement.

A different class of environmental factors includes air quality/airborne contaminants. Airborne carbon nanoparticles of the sort emitted by standard photocopiers impaired complex synaptic signaling in cortical cultures and furthermore imparted an additive impact to that of $A \beta$ at concentrations at which neither agent perturbed signaling alone [56]. Comparison of the geographical distribution of individuals with dementia or MCI as reported by the National Alzheimer's Coordinating Center with ambient air concentrations of ozone and fine particulate matter (PM) suggested that ozone but not PM was associated with more rapid cognitive decline [57]. This 
impact was confined to individuals with dementia or MCI and was not detected for individuals displaying normal cognitive performance. Individuals harboring one or more APOE4 alleles exhibited a faster rate of cognitive decline. These findings suggest that airborne contaminants are another environmental component that may influence the onset or progression of cognitive decline during dementia.

\section{TRAUMATIC BRAIN INJURY AS A RISK FACTOR FOR AD}

A growing body of evidence has linked traumatic brain injury (TBI) to subsequent manifestation of $\mathrm{AD}$ later in life [58]. TBI encompasses primary and secondary injury. Primary injury encompasses structural damage characterized by the stretching, compression, and tearing of blood vessels and tissue, the extent of which is dependent upon the nature of impact and which can damage neuronal tracts. Secondary injury encompasses an often protracted cascade of biochemical processes instigated by primary injury, resulting in excitotoxicity, cellular energy deficits, inflammation, oxidative stress, and apoptosis. Secondary injury can accumulate over time and may not be detected for days to years following the primary injury (see refs. in [59]). This is particularly troubling since socalled mild TBI can occur during relatively benign situations, including "heading" the ball during junior high school soccer. Even during a violent event, TBI can be elusive. For example, when a military vehicle runs over an explosive device, the one individual who bumps his/her head but receives no physical wound while all other do may not report for an examination. Secondary damage can therefore continue unchecked and can actually surpass the long-term damage from an overt head wound. A few years back, I stopped short at an intersection as someone ran a red light, and turned to watch the car speeding by. A jeep slammed into me from behind and pushed my car some 10-15 feet ahead, and my forehead slammed into the steering wheel. Since I was hit from behind, the airbag did not deploy, and since I had turned to watch the first car, my shoulder strap did not prevent this. The reason I include this is that $\mathrm{I}$ had absolutely no recollection of hitting my head, or even being pushed forward. However, a bruise appeared on my forehead that matched the shape of the steering wheel. How often does TBI occur leaving us unaware?

Lifestyle may influence the consequences of secondary injury. Since lifestyle modifications can also impact $\mathrm{AD}$, such modifications may be critical to reduce the incidence of development of $\mathrm{AD}$ following TBI. In initial studies to examine this possibility under controlled conditions, we induced a closed head injury (CHI) as a model of TBI in normal mice using a standardized weight drop device after which mice were housed in isolation or in groups. Cognitive performance as assayed by $\mathrm{Y}$ maze navigation was impaired in mice housed in isolation but not in groups 1-4 weeks post $\mathrm{CHI}$. CHI increased oxidative species in brain tissue from social and isolated mice, and also increased excitotoxic signaling in hippocampal slices from all mice; however, excitotoxic signaling was exacerbated by isolation [59].

These findings support the hypothesis that socialization may attenuate secondary damage following TBI. More specific AD biomarkers $(\mathrm{A} \beta$, phosphotau) were unchanged, but we recognize that we only examined these mice over a short interval following CHI. Future studies will examine the consequences of single and multiple $\mathrm{CHI}$ in normal and transgenic mice over several months in mice maintained \pm environmental, social, and dietary enrichment under the conditions described above.

With particular regard to excitotoxic signaling, neurons exposed to glutamate elaborate excitotoxic signals that differ dramatically from those elaborated by untreated control neurons. We recorded the synaptic signals elaborated by neurons treated with glutamate in our MEA system, and utilized them to stimulate additional cortical cultures. Notably, otherwise untreated neurons displayed aberrant, disorganized signaling when stimulated by a digitized synaptic signal from glutamate-treated cultures. This disorganized signaling strongly resembled that resulting from direct treatment with glutamate (unpublished observations). These findings suggest that secondary damage can spread to distal regions from a wound by synaptic activity.

\section{IS THERE A CURE?}

After all of these decades of research by so many groups, no true cure is in sight despite identification of integral processes and "at-risk" genes. However, as discussed above, while we cannot alter our genotype, we can influence our phenotype. Our studies are part of the growing body of evidence that lifestyle modifications can make a huge difference in the onset and/or progression of cognitive decline. Like diabetes or cardiovascular disease, which can be kept in check 
by lifestyle modifications, lifestyle modifications can allow an individual to delay or reduce the rate of cognitive decline such that the tipping point that warrants a diagnosis of $\mathrm{AD}$ is pushed out beyond his or her lifetime. This is, by all measures, a "functional cure" for that individual.

\section{DISCLOSURE STATEMENT}

The author's disclosure is available online (http:// j-alz.com/manuscript-disclosures/17-0724r1).

\section{REFERENCES}

[1] Smith MA, Joseph JA, Perry G (2000) Arson: Tracking the culprit in Alzheimer's disease. Ann N Y Acad Sci 924, 35-38.

[2] Shea TB, Klinger EP, Cressman CM (1995) Calcium influx recruits an additional class of kinases to hyperphosphorylate tau. Neuroreport 6, 1309-1312.

[3] Shea TB, Cressman CM (1999) The order of exposure of tau to signal transduction kinases alters the generation of "AD-like" phospho-epitopes. Cell Mol Neurobiol 19, 224235.

[4] Ekinci FJ, Shea TB (1999) Hyperactivation of mitogenactivated protein kinase increases phospho-tau immunoreactivity within human neuroblastoma: Additive and synergistic influence of alteration of additional kinase activities. Cell Mol Neurobiol 19, 249-260.

[5] Shea TB, Beermann ML, Nixon RA (1991) Multiple proteases regulate neurite outgrowth in $\mathrm{NB} 2 \mathrm{a} / \mathrm{d} 1$ neuroblastoma. J Neurochem 56, 842-851.

[6] Shea TB, Beermann ML, Spencer MA, Nixon RA (1995) Enhancement of neurite outgrowth following calpain inhibition is mediated by protein kinase C. J Neurochem $\mathbf{6 5}$, 517-527.

[7] Shea TB, Spencer MJ, Beermann ML, Cressman CM, Nixon RA (1996) Calcium influx into human neuroblastoma cells induces alz-50 immunoreactivity: Involvement of calpainmediated hydrolysis of protein kinase C. J Neurochem $\mathbf{6 6}$, 1539-1549.

[8] Shea TB, Ekinci FJ (1999) Biphasic effect of calcium influx on tau phosphorylation: Involvement of calcium-dependent phosphatases and kinases. J Alzheimers Dis 1, 353-360.

[9] Surprenant MJ, Shea TB (1998) Induction of neuronal differentiation by a mitotic inhibitor: Neuritogenesis is a default pathway invoked by withdrawal of neuroblasts from the mitotic cycle. Neurosci Res Commun 22, 59-62.

[10] Shea TB (1993) Contribution of recapitulation of developmental astroglial neuronal interactions to the neurodegeneration in Alzheimer's disease: An hypothesis. In Developmental Neurotoxicology, Harry GJ, ed. CRC Press, pp. 48-76.

[11] Shea TB (1994) Toxic and trophic effects of glial-derived factors on neuronal cultures. Neuroreport 5, 797-800.

[12] Shea TB (1995) Role of glial-derived nexin in neuronal differentiation and acute brain injury, and potential involvement in exacerbation of neurodegeneration in Alzheimer's disease. Brain Res Rev 20, 171-184.

[13] Tupper CL, Shea TB (1998) Transforming growth factor ( 2 exerts concentration-dependent protection towards and exacerbation of (-amyloid toxicity. Neurosci Res Commun 22, 83-89.

[14] Lambert MP, Stevens G, Sabo S, Barber K, Wang G, Wade W, Krafft G, Snyder S, Holzman TF, Klein WL (1994) Beta/A4-evoked degeneration of differentiated SHSY5Y human neuroblastoma cells. J Neurosci Res 39, 377-385.

[15] Ekinci FJ, Shea TB (2000) Hyperphosphorylation of tau does not correlate with neurodegeneration in cortical neurons in culture. J Alzheimers Dis 2, 7-12.

[16] Ekinci FJ, Lindsey MD, Shea TB (2000) Beta-amyloid induces neurodegeneration by oxidative stress rather than tau phosphorylation. Brain Res 76, 389-395.

[17] Shea TB, Rogers E (2002) Folate quenches oxidative damage in brains of apolipoprotein E-deficient mice: Augmentation by vitamin E. Mol Brain Res 108, 1-6.

[18] Mihalick SM, Ortiz D, Kumar R, Rogers E, Shea TB (2004) Folate and vitamin E deficiency impair cognitive performance in mice subjected to oxidative stress: Differential impact on normal mice and mice lacking apolipoprotein E. Neuromolecular Med 4, 197-202.

[19] Shea TB, Rogers E, Ortiz D, Sheu MS (2002) Apolipoprotein $\mathrm{E}$ deficiency promotes increased oxidative stress and compensatory increases in antioxidants in brain tissue. Free Rad Biol Med 33, 1115-1120.

[20] Tchantchou F, Graves M, Ashline D, Morin A, Pimenta A, Ortiz D, Rogers E, Shea TB (2004) Increased transcription and activity of glutathione synthase in response to deficiencies in folate, vitamin E and apolipoprotein E. J Neurosci Res 75, 508-155.

[21] Tchantchou F, Graves M, Rogers E, Ortiz D, Shea TB (2005) $\mathrm{N}$-acetyl cysteine alleviates neurodegeneration and increased glutathione expression in normal and ApoE- deficient mice. $J$ Alzheimers Dis 7, 135-138.

[22] Tchantchou F, Graves M, Falcone D, Shea TB (2008) S-adenosylmethionine mediates glutathione efficacy by increasing glutathione s-transferase activity: Implications for S-adenosyl methionine as a neuroprotective dietary supplement. J Alzheimers Dis 14, 323-328.

[23] Tchantchou F, Graves M, Ortiz D, Shea TB (2006) Sadenosyl methionine: A link between nutritional and genetic risk factors in Alzheimer's disease. J Nutr Health Aging 10, 541-544.

[24] Tchantchou F, Graves M, Ortiz D, Shea TB (2005) Expression and activity of methionine cycle genes are altered following folate and vitamin E deficiency under oxidative challenge: Modulation by apolipoprotein E-deficiency. Nutr Neurosci 9, 17-24.

[25] Chan A, Tchantchou F, Rogers E, Shea TB (2009) Dietary deficiency differentially regulates presenilin expression, gamma-secretase activity and Abeta levels as a function of ApoE genotype: Role of S-adenosyl methionine. J Neurochem 110, 831-836.

[26] Chan A, Tchantchou F, Graves V, Rozen R, Shea TB (2008b) Dietary and genetic compromise in folate availability reduces acetylcholine and cognitive performance: Critical role of S-adenosylmethionine. J Health Nutr Aging 12, 252-261.

[27] Ho PI, Ortiz D, Shea TB (2001) Abeta induces calcium influx via $\mathrm{L}$ voltage-sensitive calcium channels rather than NMDA channels. J Alzheimers Dis 3, 479-483.

[28] Ho PI, Ortiz D, Rogers E, Shea TB (2002) Multiple aspects of homocysteine neurotoxicity: Glutamate excitotoxicity, kinase hyperactivation and DNA damage. $J$ Neurosci Res 70, 694-702. 
[29] Ho P, Ashline D, Dhitavat S, Ortiz D, Collins SC, Rogers E, Shea TB (2003) Folate deprivation induces neurodegeneration: Roles of oxidative stress and increased homocysteine. Neurobiol Dis 14, 32-42.

[30] Ekinci FJ, Malik KM, Shea TB (1999) Beta-amyloid induces calcium influx and neurodegeneration by MAP kinase-mediated activation of the $\mathrm{L}$ voltage-sensitive calcium channel. J Biol Chem 274, 30322-30327.

[31] Tjiattas L, Ortiz D, Sirikarnt Dhivant S, Mitton K, Rogers E, Shea TB (2004) Folate deficiency and homocysteine induce toxicity in cultured dorsal root ganglion neurons via cytosolic calcium accumulation. Aging Cell 3, 71-76.

[32] Chan A, Alsaraby A, Shea TB (2008) Folate deprivation increases tau phosphorylation by homocysteine-induced calcium influx and by inhibition of phosphatase activity: Alleviation by S-adenosyl methionine. Brain Res 1199, 133137.

[33] Shea TB, Ekinci FJ, Ortiz D (2003) Okadaic acid mediates tau phosphorylation via sustained activation of the $\mathrm{L}$ voltage-sensitive calcium channel. Mol Brain Res 117, 145151.

[34] Serra M, Chan A, Dubey M, Gilman V, Shea TB (2008) Folate and $\mathrm{S}$-adenosymethionine modulate synaptic activity in cultured cortical neurons: Differential impact on normal and apolipoprotein E-deficient mice. Phys Biol 5, 44002.

[35] Shea TB, Rogers E, Remington R (2012) Nutrition and dementia: Are we asking the right questions? J Alzheimers Dis 30, 27-33.

[36] Shea TB, Remington R (2015) Nutritional supplementation for Alzheimer's disease. Curr Opin Psychiatry 28, 141-147.

[37] Chan A, Paskavitz J, Remington R, Shea TB (2008) Efficacy of a vitamin/nutriceutical formulation for early-stage Alzheimer's disease: A one-year open-label pilot study with a 16-month extension. Am J Alzheimers Dis Other Demen 23, 571-585.

[38] Remington R, Chan A, Shea TB (2009) Efficacy of a vita$\mathrm{min} /$ nutriceutical formulation for moderate to late-stage Alzheimer's disease: A placebo-controlled pilot study. Am J Alzheimers Dis Other Demen 24, 27-33.

[39] Chan A, Lepore A, Kotoya E, Zemianek J, Remington R, Shea TB (2010) Efficacy of a vitamin/nutriceutical formulation on cognitive speed and recall in adults with no known or suspected dementia. J Nutr Health Aging 14, 224-230.

[40] Shea TB, Remington R. Apparent cognitive decline as revealed by an executive function test within a cohort of elderly individuals self-reporting normal cognitive performance. $J \mathrm{Alz}$ dis, (in press).

[41] Remington R, Bechtel C, Larsen D, Lortie JJ, Samar A, Doshanjh L, Fishman P, Luo Y, Smyers K, Hoffmann H, Barysauskas C, Barton B, Shea TB (2015) A nutritional formulation for cognitive performance and mood in Alzheimer's disease: A phase II multi-site randomized trial with an open-label extension. J Alzheimers Dis 45, 395-405.

[42] Remington R, Lortie J, Hoffman H, Page R, Morrell C, Shea TB (2015) A nutritional formulation for cognitive performance in mild cognitive impairment: A placebo-controlled trial with an open-label extension. J Alzheimers Dis 48, 591-595.

[43] Remington R, Bechtel C, Larsen D, Samar A, Page R, Morrell C, Shea TB (2016) Maintenance of cognitive performance and mood for individuals with Alzheimer's disease following consumption of a nutraceutical formu- lation: A one-year, open-label study. J Alzheimers Dis 51, 991-995.

[44] Lee S, Lemere C, Frost J, Shea TB (2011) Dietary supplementation with S-adenosyl methionine delayed Abeta and tau pathology in 3xTg-AD mice. J Alzheimers Dis $\mathbf{2 8}$, 423-431.

[45] Ho PI, Dhivant S, Ashline D, Ortiz D, Rogers E, Shea TB (2001) Homocysteine potentiates amyloid-beta neurotoxicity; Role of oxidative stress. J Neurochem 78, 1-6.

[46] Lee S, Zemianek JM, Shea TB (2013) Rapid, reversible impairment of synaptic signaling in cultured cortical neurons by exogenously-applied Abeta. J Alzheimers Dis 35, 395-402.

[47] Taddeo M, Lee S, Shea TB (2014) Synergistic inhibition of synaptic signaling in cortical cultures by subcytotoxic levels of oligomerized Amyloid beta and iron: Alleviation by zinc. J Alzheimers Dis 41, 365-369.

[48] Kamboh MI (2004) Molecular genetics of late-onset Alzheimer's disease. Ann Hum Genet 68, 381-404.

[49] Parihar MS, Hemnani T (2004) Alzheimer's disease pathogenesis and therapeutic interventions. J Clin Neurosci 11, 456-467.

[50] Zawia NH, Lahiri DK, Cardozo-Pelaez (2009) Epigenetics, oxidative stress and Alzheimer's disease. Free Radic Biol Med 46, 1241-1249.

[51] Zhu X, Raina AK, Lee HG, Casadesus G, Smith MA, Perry G (2004) Oxidative stress signaling in Alzheimer's disease. Brain Res 1000, 32-39.

[52] Shea TB, Rogers E (2014) Lifetime requirement of the methionine cycle for neuronal development and maintenance. Curr Opion Psychiatry 27, 138-142.

[53] Chan A, Shea TB (2010) Apolipoprotein E3 as a risk factor for Alzheimer's disease under conditions of nutritional imbalance. J Alzheimers Dis 20, 1-8.

[54] Lee S, Doulames V, Donnelly M, Levasseau J, Shea TB (2011) Environmental enrichment can prevent cognitive decline induced by dietary oxidative challenge in mice. J Alzheimers Dis 28, 497-501.

[55] Doulames V, Lee S, Shea TB (2014) Environmental enrichment and social interaction improve cognitive function in normal adult mice. Int $J$ Neurosci 124, 369-376.

[56] Lee S, Sohal IS, Thierren M, Pal AK, Bello D, Shea TB (2015) Additive impairment of synaptic signaling in cultured cortical neurons by exogenously-applied oligomerized Abeta and airborne nanoparticles generated during photocopying. J Alzheimers Dis 47, 49-54.

[57] Galkina Cleary E, Cifuentes M, Grinstein G, Brugge D, Shea TB (2018) Association of low-level ozone with cognitive decline in older adults. J Alzheimers Dis 61, in press.

[58] Mez J, Daneshvar DH, Kiernan PT, Abdolmohammadi B, Alvarez VE, Huber BR, Alosco ML, Solomon TM, Nowinski CJ, McHale L, Cormier KA, Kubilus CA, Martin BM, Murphy L, Baugh CM, Montenigro PH, Chaisson CE, Tripodis Y, Kowall NW, Weuve J, McClean MD, Cantu RC, Goldstein LE, Katz DI, Stern RA, Stein TD, McKee AC (2017) Clinicopathological evaluation of chronic traumatic encephalopathy in players of American football. JAMA 318, 360-370.

[59] Doulames V, Vilcans M, Lee S, Shea TB (2015) Social interaction attenuates the extent of secondary neuronal damage following closed head injury in mice. Front Behav Neurosci 9, 275 . 